\title{
COMPARISON OF BREAD WHEAT (Triticum aestivum L.) LINES WITH REGISTERED CULTIVARS IN TERMS OF YIELD AND QUALITY CHARACTERISTICS
}

\author{
KARAMAN, M. ${ }^{*}-$ AKTAS, $\mathrm{H}^{2}$ \\ ${ }^{I}$ Department of Plant Production Technologies, Faculty of Applied Sciences, Mus Alparslan \\ University, Mus, Turkey \\ ${ }^{2}$ Kiziltepe Vocational School, Mardin Artuklu University, Mardin, Turkey \\ *Corresponding author \\ e-mail: karaman2178@hotmail.com; phone: +90-530-600-9136; fax: +90-436-231-2201
}

(Received 19 $9^{\text {th }}$ Dec 2019; accepted 24 $4^{\text {th }}$ Mar 2020)

\begin{abstract}
The study was carried out under rainfall conditions during the 2011-2012 and 2012-2013 growing seasons in Diyarbakir province of Turkey. The experiment had designed as a randomized block design with 3 replications. The aim of the study was to determine the lines that were superior in terms of yield and quality to the varieties. The experimental material consisted of 20 bread wheat lines and 5 standard varieties. According to the results of variance analysis; significant differences were observed between genotypes in all features at $1 \%$. Grain yield $(\mathrm{GY})$ had a significant positive relationship with test weight (TW), and a significant negative relationship with wet gluten (WG). Also, grain hardness (GH) (PSI: Particle size index) had significant negative relationship with TW, and a significant positive relationship with plant height $(\mathrm{PH})$ and heading time (HT). 4 lines in grain yield, 3 lines in test weight, 1 line in protein content, 4 lines in zeleny sedimentation and 1 line in wet gluten showed superior performance than all standards. It is concluded that the G19 and G24 lines may be candidates for registration.
\end{abstract}

Keywords: correlation, grain hardness, protein content, wet gluten

\section{Introduction}

Wheat has been a main source of nutrient throughout the ages, and it continues to strengthen its role in human life. 23.4 million hectare of agricultural land is cultivated in Turkey, It is reported that the area allocated for agricultural activities except fallow areas is $66.4 \%$. Also, it is reported that cereal sowing amount has $71 \%$ share in this area and wheat cultivation takes first place among cereals in proportion (TSI, 2018).

The center of origin of wheat in the world and also the first place where wheat and barley cultivation is made is called fertile crescent. Southeast Anatolia Region is located in fertile crescent region. So, of Turkey it is considered to have a significant track record in wheat breeding and commissioning culture of wheat (Civgın, 2016; Aktas, 2017).

Central Anatolia Region in wheat production in Turkey takes first place with rate of $33.5 \%$. Southeastern Anatolia Region ranks second in wheat production with a rate of $16.5 \%$ (TSI, 2018). Diyarbakir is one of the important provinces of Southeastern Anatolia Region in wheat production. 1.1 million tons of wheat is produced in Diyarbakir. This amount constitutes $3.5 \%$ of the total wheat production in Turkey (TSI, 2018). The annual per capita wheat consumption in Turkey is over $200 \mathrm{~kg}$. Due to the high daily consumption per capita and being one of the most important caloric sources of Turkish people, wheat maintains its strategic importance (Morgounov et al., 2016).

It has been reported that the amount of protein in the grain is affected significantly by environmental conditions (climate, fertilizer amount, irrigation, etc.) and also the 
inheritance factor is an important determinant on quality parameters in wheat grown in both irrigated and rainfall conditions (Grausgruber et al., 2000; Souza et al., 2004).

It is emphasized that the amount of protein in bread wheat is important in determining quality. However, since the amount of protein alone is not sufficient, it has been reported that the amount of sedimentation and gluten should be taken into account (Gooding et al., 2003). Plant breeders aim to develop genotypes with high grain yield, quality and ability to adapt to different environments (Kendal and Sener, 2015).

With this purpose, study was conducted in Diyarbakir conditions in Turkey. After comparing the wheat lines of abroad origin with registered varieties in terms of yield and quality characteristics, the lines that can be high performance candidates were determined.

\section{Materials and methods}

The study was carried out under rainfall conditions in Diyarbakir province of Turkey in 2011-2012 and 2012-2013 growing seasons with 5 standard varieties and 20 advanced bread wheat lines (Fig. 1; Table 1).

Table 1. Origins of bread wheat genotypes used in the research

\begin{tabular}{c|c|c|c}
\hline Genotypes (G) & Character & Pedigree & Origin \\
\hline G1 & Spring & Attila-7 (Check) Ndvg9144//Kal/Bb/37yaco/4/Vee\#5 Cm85836... & CIMMYT \\
G2 & Spring & Nadia-15 Van's'/3/Cndr's/Ana//Cndr's/Mus's/4/Tevee-5 Icw99... & CIMMYT \\
G3 & Spring & Sisaban-4shuha-5/Asfoor-1 Icw00-0207-0ap-0ap-0ap-56ap-0ap... & CIMMYT \\
G4 & Spring & Morsud-22bt1735//Achtar//Hubara-8 Icw01-00162-0ap-3ap-0ap.. & CIMMYT \\
Nurkent & Spring & Check & GAP UTAEM \\
G6 & Spring & Qafzah-14/Asfoor-1 Icw01-00223-0ap-11ap-0ap-0ap-1ap-0ap... & CIMMYT \\
G7 & Spring & Usher-16 Crow's'/Bow's'-1994/95//Asfoor-5 Icw01-00257-0ap... & CIMMYT \\
G8 & Spring & Pastor//Hx17573/2*Bau/3/Sokoll/Wbll1 Ptss02b00098t-0topy... & CIMMYT \\
G9 & Spring & Croc_1/Ae.Squarrosa(213)//Pgo/3/Cmh81.38/2*Kauz/4/Berkut... & CIMMYT \\
Pehlivan & Winter & Check & TTAEM \\
G11 & Spring & Croc_1/Ae.Squarrosa(213)//Pgo/3/Cmh81.38/2*Kauz/4/Berkut... & CIMMYT \\
G12 & Spring & Chen/Aeg1lops Squarrosa (Taus)//Bcn/3/Bav92/4/Berkut Cmsa... & CIMMYT \\
G13 & Spring & Ser1//Au/Up301/3/Je93 7.20/4/Milan/Amsel See02528-0s-0s-0sd. & CIMMYT \\
G14 & Spring & Opata*2/Wulp/3/Sara1/Yaco//Att1la/4/Har 1685 See02556-0tops. & CIMMYT \\
Cemre & Spring & Check & GAP UTAEM \\
G16 & Spring & V763.2312/V879.C8.11.11/Sn.64/Hn.4//Rex/3/Edch/Mex/4/... & CIMMYT \\
G17 & Spring & Basribey/3/Agri/Nacozari-Sd... & CIMMYT \\
G18 & Spring & Tam200/Pastor//Toba97Cmss99y02667t-060m-040y-040m... & CIMMYT \\
G19 & Spring & Oasis/Skauz//4*Bcn/3/Pastor/4/Kauz*2/Yaco//Kauzcmss... & CIMMYT \\
Sagittario & Alternative & Check & TASACO TARM. \\
G21 & Spring & Qafzah-31(Check) Sha5//Carc/Auk/3/Vee//5//Dobuc's Cmss93... & CIMMYT \\
G22 & Spring & Misket-12-Bti735/Achtar//Asfoor-1 Icw01-00164-0ap-1ap-0ap... & CIMMYT \\
G23 & Spring & Rebwah-12/Zemamra-8-Rebwah-12/Zemamra-8 Icw01-00193... & CIMMYT \\
G24 & Spring & Qafzah-14/Asfoor-1-Qafzah-14/Asfoor-1 Icw-0100223-0ap... & CIMMYT \\
Adana-99 & Spring & Check & DATAE \\
\hline
\end{tabular}

G: Genotypes, CIMMYT: International Maize and Wheat Improvement Center, GAP UTAEM: GAP International Agricultural Research and Training Center, DATAE: Eastern Mediterranean Agricultural Research Institute, TASACO TARM.: Tasaco Agriculture, TTAEM: Directorate of Trakya Agricultural Research Institute 


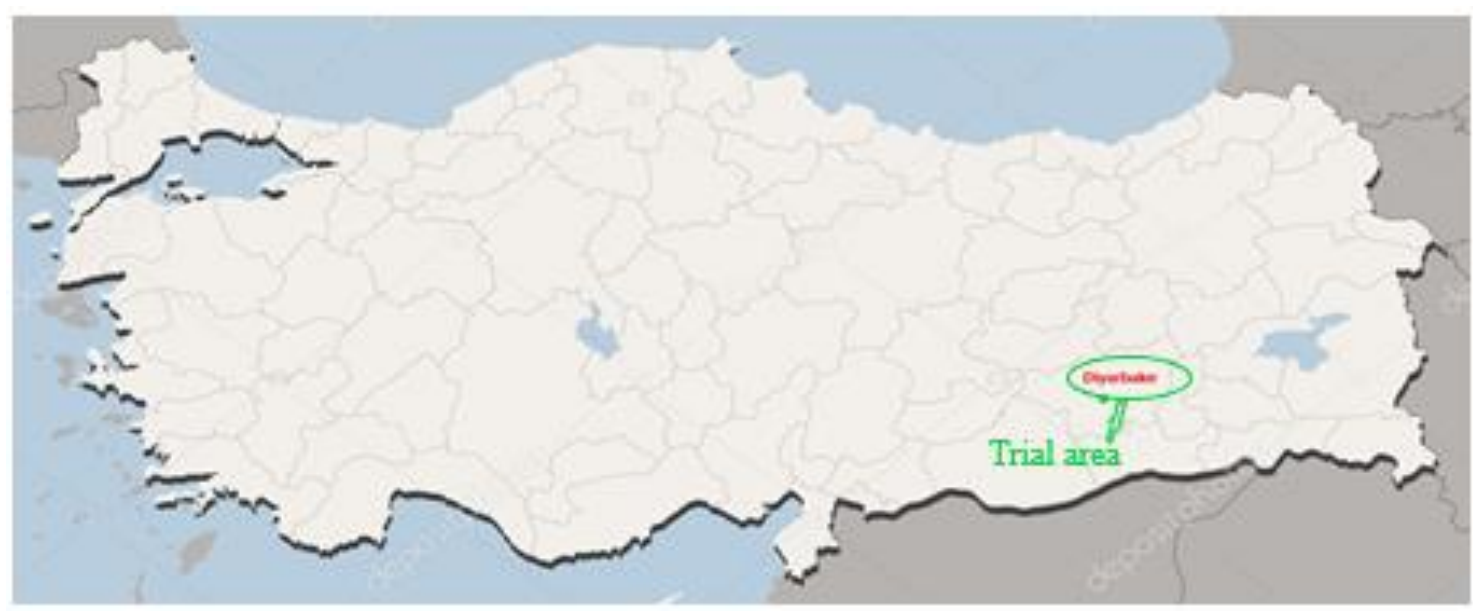

Figure 1. Experiment location's place in the Turkey map

In the first year of the study, rainfall was below the average of long years, while in the second year there was rainfall above the average of long years (Fig. 2).

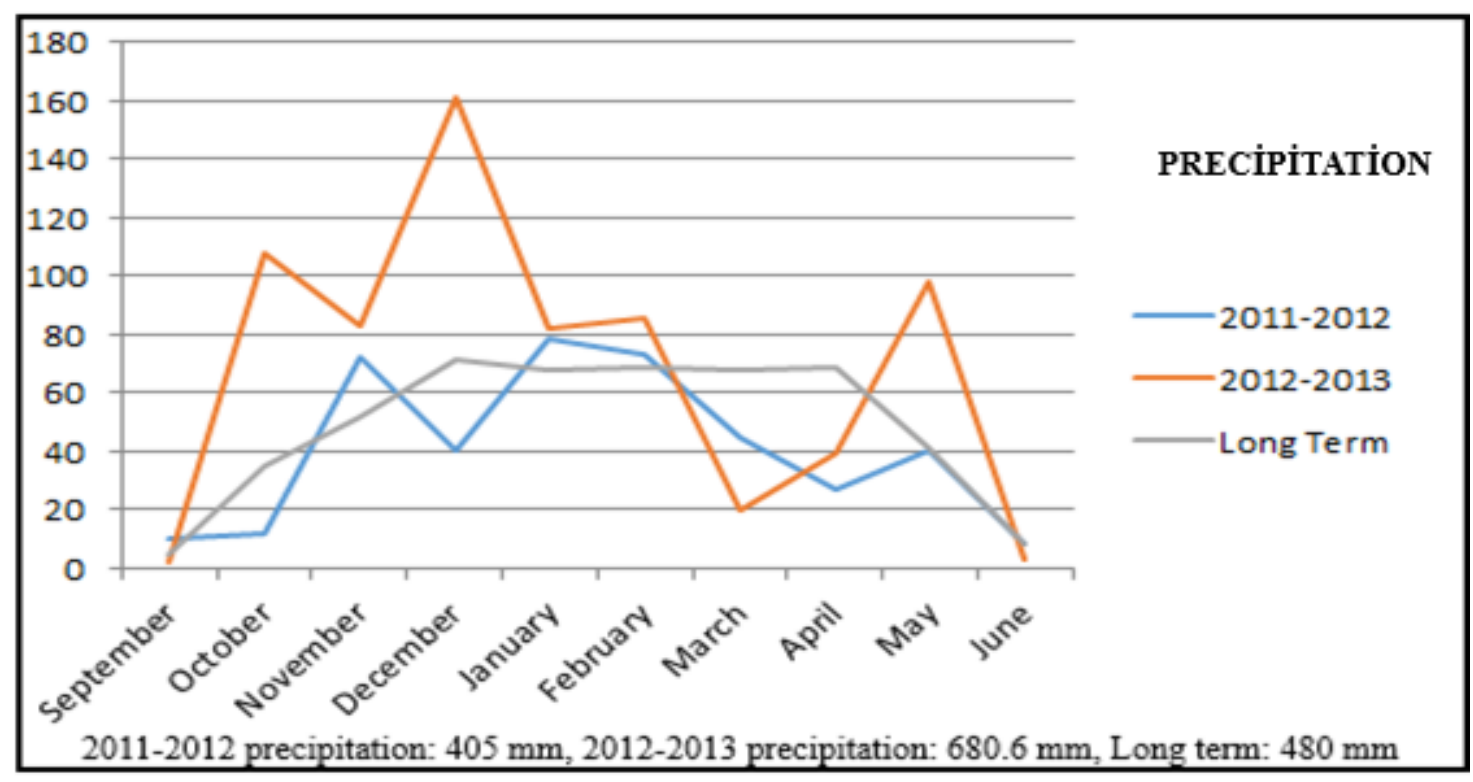

Figure 2. The amount of precipitation of Diyarbakir's in 2011-2012 and 2012-2013 growing seasons and long terms $(\mathrm{mm})$

However, it was determined that the amount of precipitation was not reflected to the grain yield at the expected level since the distribution of rainfall was irregular on months basis.

In the first year of the study, the average temperatures based on months were below long years. However, the second year has been more than long years (Fig. 3).

The experiment was conducted in randomized block design with 3 replications. All of the lines used in the study are of spring nature. Additionally, Pehlivan winter, Sagittario alternative, Nurkent, Cemre and Adana-99 varieties are of spring character. 450 seeds were sowed in the square meter and the size of the parcel was $6 \mathrm{~m}^{2}$. 


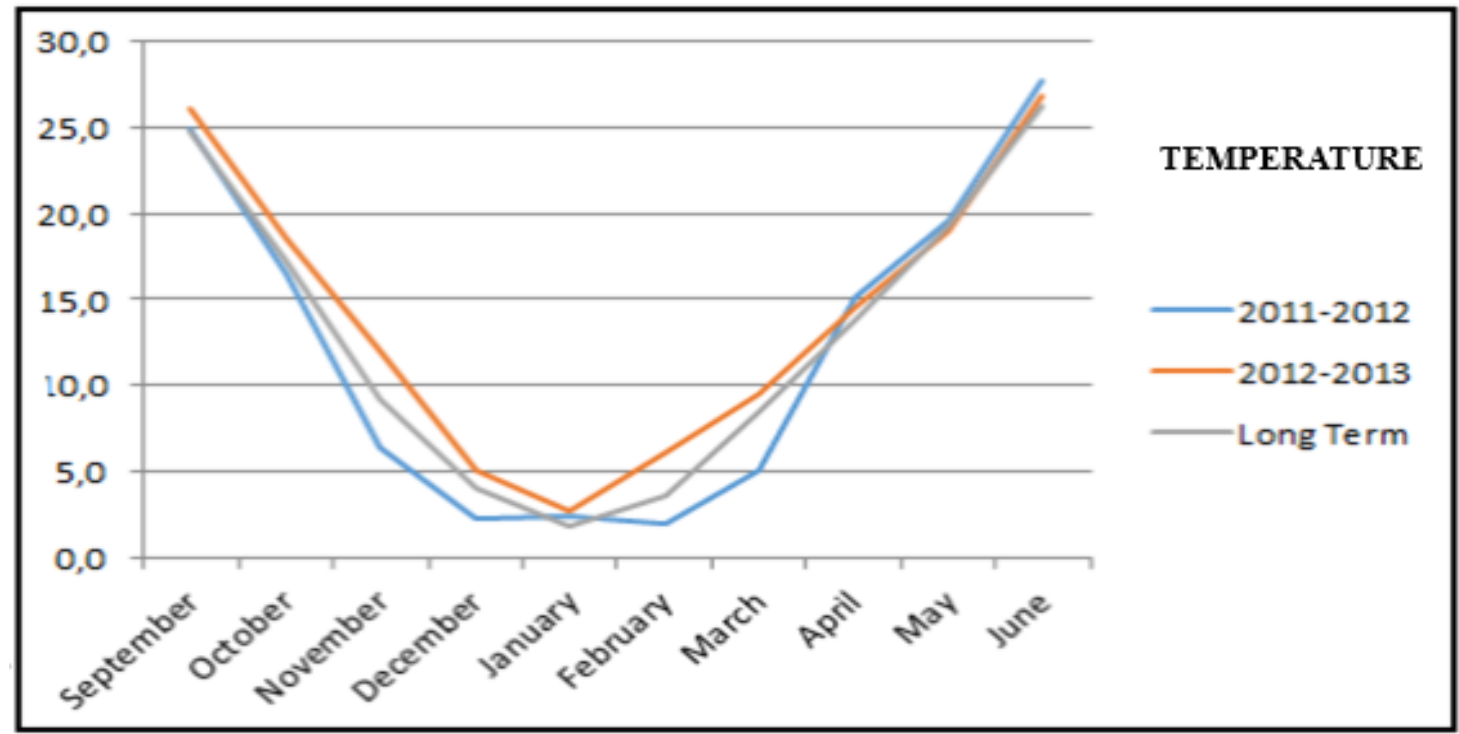

Figure 3. Average temperature of Diyarbakir's in 2011-2012 and 2012-2013 growing seasons and long terms $\left({ }^{\circ} \mathrm{C}\right)$

The amount of fertilizer to be used in the experiment was calculated over $120 \mathrm{~kg} \mathrm{ha}^{-1}$ pure nitrogen $(\mathrm{N})$ and $60 \mathrm{~kg} \mathrm{ha}^{-1}$ pure phosphorus $\left(\mathrm{P}_{2} \mathrm{O}_{5}\right)$. Half of the total nitrogen and all of the phosphorus were used with sowing and the remaining half of the nitrogen was given at the end of the tillering period. Experiment was sowed in the first week of November and harvesting was done with the combine harvester in the second week of June. The study area is $37^{\circ} 56$ north latitude and $43^{\circ} 15$ east longitude and has a height of $599 \mathrm{~m}$. It is determined that the soil formation class is clayey, the salt content is $0.25 \%$, PH: 7.8 , the amount of organic matter is $0.68 \%$ and the saturation rate with water is $77 \%$. (Anonymous, 2013).

In the study, heading time (HT) was determined as the number of days as of January 1, taking into account the date when $50 \%$ of the plants in each parcel were spiked. When determining plant height (PH), 10 plants were randomly selected per plot. Then, the length from soil level to the highest spikelet except for awns was measured in $\mathrm{cm}$. Grain yield (GY) was determined by turning the yield in $\mathrm{kg} \mathrm{ha}^{-1}$ after the whole parcel was harvested. When determining the thousand grain weight (TGW), 100 seeds were counted 4 times and weighed on a 0.01 precision scale, then averaged and multiplied by 10 and determined in grams.

To determine the test weight (TW), the seed obtained from each parcel after harvest was weighed after being put into a 1-L container and its weight was multiplied by 100 . Protein content (PR) was determined with the Near Infrared Model 6500 device according to the AACC 39-10 method (Anonymous, 1990). Amount of zeleny sedimentation (ZS) ICC-No. according to the 115 was determined (Anonymous, 1982). Wet gluten (WG) ratio was determined by the Glutomatic 2200 device according to ICC standard 155/1 method (Anonymous, 1994). Grain hardness (GH) (Particle size index) was made according to the AACC 39-70 A method (Anonymous, 2000). In the research, variance analysis of the data was performed with JMP 13.0 statistical package program. Differences in mean values were examined by LSD test $(\mathrm{p} \leq 0.01$ or $\mathrm{p} \leq 0.05)$ (Gomez and Gomez, 1984). 


\section{Results and discussion}

According to the results of ANOVA analysis, of squares mean and significance levels of the investigated features are given in Tables 2 and 3. In terms of other features, except for grain yield (n.s.) and zeleny sedimantasyon (5\%), between years were observed significant differences at the level of $1 \%$. In addition, except for grain hardness, it is seen that the responses ( $\mathrm{YxG}$ ) of genotypes by years are different from each other (Tables 2 and 3).

Table 2. Mean of squares according to variance analysis results

\begin{tabular}{c|c|c|c|c|c|c}
\hline $\begin{array}{c}\text { Variance } \\
\text { resources }\end{array}$ & $\begin{array}{c}\text { Degree of } \\
\text { freedom }\end{array}$ & $\begin{array}{c}\text { Heading } \\
\text { time }\end{array}$ & $\begin{array}{c}\text { Plant } \\
\text { height }\end{array}$ & $\begin{array}{c}\text { Grain } \\
\text { yield }\end{array}$ & $\begin{array}{c}\text { Test } \\
\text { weight }\end{array}$ & $\begin{array}{c}\text { Thousand } \\
\text { grain weight }\end{array}$ \\
\hline $\mathrm{Y}$ & 1 & $3591.7^{* *}$ & $8816.6^{* *}$ & $994762.5 \mathrm{n} . \mathrm{s}$. & $77.3^{* *}$ & $518.1^{* *}$ \\
$\mathrm{R}$ & 4 & $12.0^{* *}$ & $110.9^{* *}$ & $1695965.4 * *$ & $2.5^{* *}$ & $4.1 \mathrm{n} . \mathrm{s}$ \\
$\mathrm{G}$ & 24 & $28.2^{* *}$ & $228.5^{* *}$ & $1007080.0^{* *}$ & $8.5^{* *}$ & $19.9 * *$ \\
$\mathrm{Y} * \mathrm{G}$ & 24 & $19.7^{* *}$ & $101.6^{* *}$ & $1330460.1 * *$ & $9.5^{* *}$ & $36.4^{* *}$ \\
\hline $\mathrm{CV}(\%)$ & & 0.7 & 1.8 & 9.5 & 0.7 & 8.2 \\
\hline
\end{tabular}

**: 0.01 level, *: 0.05 level statistically significant, Y: Year, R: Replication, G: Genotype, Y* G:: Year

* Genotype interaction, n.s.: not significant

Table 3. Mean of squares according to variance analysis results

\begin{tabular}{c|c|c|c|c|c}
\hline $\begin{array}{c}\text { Variance } \\
\text { resources }\end{array}$ & $\begin{array}{c}\text { Degree } \\
\text { of freedom }\end{array}$ & $\begin{array}{c}\text { Protein } \\
\text { content }\end{array}$ & $\begin{array}{c}\text { Zeleny } \\
\text { sedimantasyon }\end{array}$ & $\begin{array}{c}\text { Wet } \\
\text { gluten }\end{array}$ & $\begin{array}{c}\text { Grain hardness } \\
\text { (PSI) }\end{array}$ \\
\hline $\mathrm{Y}$ & 1 & $77.6^{* *}$ & $468.8^{*}$ & $141.7^{* *}$ & $949.0^{* *}$ \\
$\mathrm{R}$ & 4 & $0.2 \mathrm{n} . \mathrm{s}$. & $48.9^{* *}$ & $1.1 \mathrm{n} . \mathrm{s}$. & $3.9 \mathrm{n} . \mathrm{s}$. \\
$\mathrm{G}$ & 24 & $0.5 * *$ & $51.6^{* *}$ & $4.7 * *$ & $134.0^{* *}$ \\
$\mathrm{Y} * \mathrm{G}$ & 24 & $0.6^{* *}$ & $30.6^{* *}$ & $6.4 * *$ & 0.07 n.s. \\
\hline $\mathrm{CV}(\%)$ & & 2.9 & 10.3 & 4.1 & 4.1 \\
\hline
\end{tabular}

In the study, the average values of heading time are given in Table 4. The average experiment of heading time was 119.4 days. While the early heading genotypes were G14 (115.3 days) and G22 (115.3 days), in terms of heading time means, Pehlivan (124.0 days) was the late heading genotype. In terms of heading time, it was determined that all lines were earlier heading than the earliest heading standard cultivar (Adana-99; 121.2 days).

Because temperature stress is an important problem in the Southeastern Anatolia Region, early heading genotypes not exposed to heat stress are preferred. In this case, early heading varieties rather than late heading varieties are in demand (Tekdal and Yildirim, 2017). In the selection study, early heading and medium early heading varieties were selected. Kiseleva and Salina (2018) reported that many genes interact for determination of heading time in bread wheat. Also, they emphasized that the genes that affect the heading time of wheat are not studied sufficiently, but the number of studies is increasing day by day.

Average plant height was determined as $99.4 \mathrm{~cm}$. Cemre cultivar had the longest plant length with $110.8 \mathrm{~cm}$ and Sagittario cultivar had the shortest plant height with $80.8 \mathrm{~cm}$ (Table 4). In studies conducted on wheat under different conditions, it was 
determined that heredity had a significant effect on plant height. In addition, climate, soil structure and agronomical application (fertilization, soil processing, etc.) has been reported to be effective on plant height (Caierao, 2006).

Table 4. Statistical significance groups for heading time, plant height and grain yield

\begin{tabular}{|c|c|c|c|c|c|c|c|c|c|}
\hline \multirow{2}{*}{ Genotypes } & \multicolumn{3}{|c|}{ HT (day) } & \multicolumn{3}{|c|}{ PH (cm) } & \multicolumn{3}{|c|}{ GY $\left(\mathrm{kg} \mathrm{ha}^{-1}\right)$} \\
\hline & 1. year & 2. year 1 & Av. & 1. year & 2. year & Av. & 1 year & 2. year & Av. \\
\hline G1 & $124.0^{\mathrm{f}}$ & $113.7^{\mathrm{gh}}$ & $118.8^{\text {fh }}$ & $97.7^{\mathrm{f}}$ & $96.0^{b-d}$ & $96.8^{\mathrm{j}-\mathrm{k}}$ & $7258^{\mathrm{ab}}$ & $7338^{a-d}$ & $7298^{\mathrm{ab}}$ \\
\hline $\mathrm{G} 2$ & $125.0^{\mathrm{e}}$ & $114.0^{\mathrm{f}-\mathrm{h}}$ & $119.5^{\mathrm{e}-\mathrm{g}}$ & $102.7^{\mathrm{e}}$ & $100.0^{\mathrm{a}}$ & $101.3^{\mathrm{f}-1}$ & $6148^{\text {b-e }}$ & $6272^{\mathrm{fg}}$ & $6210^{g-h}$ \\
\hline G3 & $123.8^{\mathrm{f}}$ & $117.9^{a b c}$ & $120.9^{\mathrm{cd}}$ & $95.7^{\mathrm{f}}$ & $99.7^{a b c}$ & $97.7^{j}$ & $6096^{\text {cde }}$ & $6474^{\mathrm{d}-\mathrm{g}}$ & $6285^{\mathrm{fgh}}$ \\
\hline G4 & $124.0^{\mathrm{f}}$ & $115.0^{\mathrm{d}-\mathrm{g}}$ & $119.5^{\mathrm{e}-\mathrm{g}}$ & $107.7^{\mathrm{d}}$ & $92.7^{\mathrm{def}}$ & $100.2^{\mathrm{h} 1}$ & $6558^{a b c}$ & $6280^{\mathrm{fg}}$ & 6419 efg \\
\hline Nurkent & $127.0^{\mathrm{d}}$ & $118.3^{\mathrm{ab}}$ & $122.7^{b}$ & $117.5^{b}$ & $97.9^{a b c}$ & $107.7^{b}$ & $6104^{\text {cde }}$ & $7188^{\text {b-f }}$ & $6646^{b g}$ \\
\hline G6 & $121.0^{1}$ & $116.7^{\mathrm{bcd}}$ & $118.8^{\mathrm{f}-\mathrm{h}}$ & $112.7^{\mathrm{c}}$ & $87.7^{\text {h }}$ & $100.2^{\mathrm{hr}}$ & $6992^{a b c}$ & $7291^{\mathrm{a}-\mathrm{e}}$ & $7142^{a-e}$ \\
\hline G7 & $120.0^{\mathrm{j}}$ & $115.7^{\text {def }}$ & $117.8^{\mathrm{ij}}$ & $97.5^{\mathrm{f}}$ & $88.5^{\mathrm{gh}}$ & $93.0^{\mathrm{lm}}$ & $7028^{a b c}$ & $5875^{\mathrm{g}}$ & $6452^{d-g}$ \\
\hline G8 & $122.5^{\mathrm{g}}$ & $114.8^{\mathrm{e}-\mathrm{g}}$ & $118.7^{\mathrm{g}-1}$ & $111.7^{\mathrm{c}}$ & $92.7^{\mathrm{e}-\mathrm{g}}$ & $102.2^{\mathrm{e}-\mathrm{h}}$ & $7562^{a}$ & $6349^{\mathrm{d}-\mathrm{g}}$ & $6956^{a-f}$ \\
\hline G9 & $122.0^{\mathrm{h}}$ & $114.3^{\mathrm{e}-\mathrm{g}}$ & $118.2^{\mathrm{h}-\mathrm{j}}$ & $112.2^{\mathrm{c}}$ & $88.8^{\text {gh }}$ & $100.5^{\mathrm{g}_{1}}$ & $6689^{a b c}$ & $6327^{\text {efg }}$ & $6508^{c-f}$ \\
\hline Pehlivan & $129.0^{\mathrm{b}}$ & $119.0^{\mathrm{a}}$ & $124.0^{\mathrm{a}}$ & $112.6^{\mathrm{c}}$ & $95.1^{\mathrm{cde}}$ & $103.9 \mathrm{de}$ & $6737 \mathrm{abc}$ & $7069^{\mathrm{b}-\mathrm{f}}$ & $6903^{a-g}$ \\
\hline G11 & $122.0^{\mathrm{h}}$ & $114.3^{\mathrm{e}-\mathrm{g}}$ & $118.2^{\mathrm{h}-\mathrm{j}}$ & $112.4^{\mathrm{c}}$ & $93.6^{\mathrm{de}}$ & $103.0^{\text {ef }}$ & $7019^{a b c}$ & $6547^{\mathrm{d}-\mathrm{g}}$ & $6783^{a-g}$ \\
\hline G12 & $122.0^{\mathrm{h}}$ & $114.7^{\mathrm{e}-\mathrm{g}}$ & $118.3^{\mathrm{h}-\mathrm{j}}$ & $112.8^{c}$ & $94.2^{\text {cde }}$ & $103.5^{\mathrm{de}}$ & $7178^{a b c}$ & $5858^{\mathrm{g}}$ & $6518^{\mathrm{c}-\mathrm{g}}$ \\
\hline G13 & $121.0^{1}$ & $114.3^{\mathrm{e}-\mathrm{g}}$ & $117.7^{\mathrm{j}}$ & $117.6^{b}$ & $92.8^{\text {def }}$ & $105.2^{\mathrm{cd}}$ & $6866^{a b c}$ & $6452^{\mathrm{d}-\mathrm{g}}$ & $6659^{b-g}$ \\
\hline G14 & $117.3^{\mathrm{k}}$ & $113.3^{\mathrm{g}-\mathrm{h}}$ & $115.3^{\mathrm{k}}$ & $112.3^{c}$ & $92.3^{\text {def }}$ & $102.3^{\mathrm{e}-\mathrm{g}}$ & $7444^{\mathrm{a}}$ & $7127^{\text {b-f }}$ & $7286^{a b}$ \\
\hline Cemre & $130.0^{\mathrm{a}}$ & $116.7^{\mathrm{bcd}}$ & $123.3^{\mathrm{ab}}$ & $122.7^{\mathrm{a}}$ & $99.0^{\mathrm{ab}}$ & $110.8^{a}$ & $6558^{a b c}$ & $7816^{\mathrm{ab}}$ & $7187^{a-d}$ \\
\hline G16 & $125.0^{\mathrm{e}}$ & $112.3^{\mathrm{h}}$ & $118.7^{\mathrm{g}-1}$ & $102.4^{\mathrm{e}}$ & $89.3^{\mathrm{f}-\mathrm{h}}$ & $95.9^{\mathrm{jk}}$ & 5138 de & $8227^{a}$ & $6683^{\mathrm{bg}}$ \\
\hline G17 & $125.0^{\mathrm{e}}$ & $114.3^{\mathrm{e}-\mathrm{g}}$ & $119.7^{\text {e-f }}$ & $97.9^{\mathrm{f}}$ & $87.5^{h}$ & $92.7^{\mathrm{m}}$ & $6508^{a b c}$ & $6636^{\mathrm{d}-\mathrm{g}}$ & $6572^{\mathrm{bg}}$ \\
\hline G18 & $124.0^{\mathrm{f}}$ & $113.3^{\mathrm{gh}}$ & $118.7^{\mathrm{g}-1}$ & $97.4^{\mathrm{f}}$ & $92.6^{\mathrm{def}}$ & $95.0^{\mathrm{kl}}$ & $7242^{a b}$ & $6669^{\mathrm{c}-\mathrm{g}}$ & $6955^{a-f}$ \\
\hline G19 & $124.0^{\mathrm{f}}$ & $113.7^{\mathrm{gh}}$ & $118.8^{\mathrm{f}-\mathrm{h}}$ & $102.3^{\mathrm{e}}$ & $87.3^{\mathrm{h}}$ & $94.8^{\mathrm{kl}}$ & $6672^{a b c}$ & $7811^{a b}$ & $7242^{a b c}$ \\
\hline Sagittario & $130.0^{\mathrm{a}}$ & $116.0^{\mathrm{c}-\mathrm{e}}$ & $123.0^{\mathrm{b}}$ & $82.7 \mathrm{~g}$ & $79.0^{1}$ & $80.8^{n}$ & $5083^{\mathrm{e}}$ & $6230 \mathrm{fg}$ & $5657^{\mathrm{h}}$ \\
\hline $\mathrm{G} 21$ & $128.0^{c}$ & $108.3^{1}$ & $118.2^{\mathrm{h}-\mathrm{j}}$ & $97.3 \mathrm{f}$ & $89.0^{\mathrm{f}-\mathrm{h}}$ & $93.2^{\mathrm{lm}}$ & $6514^{a b c}$ & $6805^{\mathrm{c}-\mathrm{g}}$ & $6660^{b-g}$ \\
\hline $\mathrm{G} 22$ & $123.3^{\mathrm{fg}}$ & $107.3^{1}$ & $115.3^{\mathrm{k}}$ & $108.3 \mathrm{~d}$ & $81.7^{1}$ & $95.0^{\mathrm{kl}}$ & $7014^{a b c}$ & $6730^{\mathrm{c}-\mathrm{g}}$ & $6872^{a-g}$ \\
\hline $\mathrm{G} 23$ & $125.2^{\mathrm{e}}$ & $114.8^{\mathrm{e}-\mathrm{g}}$ & $120.0^{\mathrm{de}}$ & $112.3 \mathrm{c}$ & $87.7^{\text {h }}$ & $100.0^{1}$ & $6211^{\mathrm{b}-\mathrm{d}}$ & $7058^{\text {b-f }}$ & $6635^{b-g}$ \\
\hline G24 & $123.3^{\mathrm{fg}}$ & $114.3^{\mathrm{e}-\mathrm{g}}$ & $118.8^{\mathrm{f}-\mathrm{h}}$ & $112.2 \mathrm{c}$ & $100.5^{\mathrm{a}}$ & $106.4^{b c}$ & $7239^{a b}$ & $7658^{a b c}$ & $7449^{a}$ \\
\hline Adana-99 & $126.7^{\mathrm{d}}$ & $115.7^{\mathrm{d}-\mathrm{f}}$ & $121.2^{c}$ & $112.7 \mathrm{c}$ & $92.3^{\text {def }}$ & $102.5^{\mathrm{e}-\mathrm{g}}$ & $7225^{a b}$ & $7069^{\text {b-f }}$ & $7147^{\mathrm{a}-\mathrm{e}}$ \\
\hline Average & 124.0 & 114.5 & 119.4 & 107.0 & 91.7 & 99.4 & 6683 & 6846 & 6765 \\
\hline Passed of check & 19 & 17 & 20 & 0 & 0 & 0 & 5 & 1 & 4 \\
\hline $\operatorname{LSD}(0.05)$ & $0.7 * *$ & $1.9 * *$ & $1.0 * *$ & $1.3 * *$ & $3.9 * *$ & $2.1 * *$ & $1120 * *$ & $990.0 * *$ & $738.1 * *$ \\
\hline
\end{tabular}

In terms of grain yield, the experiment average was $6765 \mathrm{~kg} \mathrm{ha}^{-1}$ and G24 $\left(7449 \mathrm{~kg} \mathrm{ha}^{-1}\right)$ line had the highest grain yield. In terms of grain yield, 4 lines passed the standard variety with the highest grain yield (Cemre; $\left.7187 \mathrm{~kg} \mathrm{ha}^{-1}\right)$ (Table 4). It has been reported that grain yield and quality parameters in wheat are generally affected by genotypes, environmental conditions and genotype environment interaction. (Barutcular et al., 2016; Kizilkeci, 2019).

The average experiment for test weight was $81.7 \mathrm{~kg} \mathrm{hl}^{-1}$. In addition, G14 $\left(83.1 \mathrm{~kg} \mathrm{hl}^{-}\right.$ $\left.{ }^{1}\right)$, G18 $\left(83.1 \mathrm{~kg} \mathrm{hl}^{-1}\right)$ and $\mathrm{G} 21\left(83.1 \mathrm{~kg} \mathrm{hl}^{-1}\right)$ lines by share the same group and were the highest test weight (Table 5). In terms of test weight, 3 lines showed higher performance than the highest standard variety (Adana-99; $83.0 \mathrm{~kg} \mathrm{hl}^{-1}$ ) (Table 5). In a 
study conducted on bread wheat in India conditions, it was reported that the weight of test ranged from 70.0 to $84.6 \mathrm{~kg} \mathrm{hl}^{-1}$. In this study, the results were similar since the average test weight was $81.7 \mathrm{~kg} \mathrm{hl}^{-1}$ (Panghal et al., 2019).

Table 5. Test weight, thousand grain weight and protein content values obtained

\begin{tabular}{|c|c|c|c|c|c|c|c|c|c|}
\hline \multirow{2}{*}{ Genotypes } & \multicolumn{3}{|c|}{ TW $\left(\mathrm{kg} \mathrm{hl}^{-1}\right)$} & \multicolumn{3}{|c|}{ TGW (g) } & \multicolumn{3}{|c|}{ PR (\%) } \\
\hline & 1. year & 2. year & Av. & 1. year & 2. year & Av. & 1. year & 2. year & Av. \\
\hline G1 & $82.1^{\text {de }}$ & $82.6^{\mathrm{b}-\mathrm{g}}$ & 82.4 bcd & $28.4^{1}$ & $45.6^{a}$ & $37.0^{\mathrm{a}-\mathrm{d}}$ & $13.8^{\mathrm{h}-\mathrm{j}}$ & $12.9^{\mathrm{a}-\mathrm{e}}$ & $13.3^{\mathrm{d}-\mathrm{h}}$ \\
\hline G2 & $79.2^{\mathrm{ij}}$ & $82.0^{\mathrm{f}-\mathrm{k}}$ & $80.6^{\text {gh }}$ & $29.6^{\mathrm{h} 1}$ & $39.5^{\mathrm{bcd}}$ & $34.6^{\mathrm{c}-\mathrm{g}}$ & $13.8^{\mathrm{g}-\mathrm{j}}$ & $13.1^{\mathrm{a}-\mathrm{d}}$ & $13.5^{\mathrm{cg}}$ \\
\hline G3 & $77.6^{\mathrm{lm}}$ & $81.9^{\mathrm{f}-\mathrm{k}}$ & $79.8^{\mathrm{ij}}$ & $34.3^{\mathrm{b}-\mathrm{g}}$ & $38.3^{b-f}$ & $36.3^{\mathrm{b}-\mathrm{f}}$ & $14.7^{b c}$ & $13.1^{\mathrm{a}-\mathrm{d}}$ & $13.9^{a b c}$ \\
\hline G4 & $83.5^{\mathrm{ab}}$ & $81.8^{\mathrm{g}-\mathrm{k}}$ & $82.6^{\mathrm{abc}}$ & $32.5^{\mathrm{f}-1}$ & $34.0^{\mathrm{e}-\mathrm{f}}$ & $33.3^{\mathrm{fg}}$ & $14.1^{\mathrm{e}-\mathrm{h}}$ & $13.0^{\mathrm{a}-\mathrm{e}}$ & $13.5^{\mathrm{c}-\mathrm{g}}$ \\
\hline Nurkent & $76.2^{n}$ & $83.5^{\mathrm{abc}}$ & $79.9^{\mathrm{ij}}$ & $33.4^{\mathrm{d}-\mathrm{h}}$ & $35.4^{\text {def }}$ & $34.4^{\mathrm{c}-\mathrm{g}}$ & $14.2^{\mathrm{c}-\mathrm{h}}$ & $12.6^{\mathrm{c}-\mathrm{f}}$ & $13.4^{\mathrm{d}-\mathrm{h}}$ \\
\hline G6 & $82.5^{\mathrm{cd}}$ & $83.3^{\mathrm{a}-\mathrm{d}}$ & $82.9^{\mathrm{ab}}$ & $31.6^{\mathrm{f}-1}$ & $33.9^{e-f}$ & $32.8^{\mathrm{g}}$ & $14.2^{\mathrm{c}-\mathrm{h}}$ & $12.5^{\mathrm{c}-\mathrm{f}}$ & $13.4^{\mathrm{d}-\mathrm{h}}$ \\
\hline G7 & $77.8^{\mathrm{lm}}$ & $82.5^{\mathrm{c}-\mathrm{h}}$ & $80.2^{\text {hı }}$ & $34.3^{\mathrm{b}-\mathrm{g}}$ & $36.8^{\mathrm{c}-\mathrm{f}}$ & $35.5^{\mathrm{c}-\mathrm{g}}$ & $14.3^{\mathrm{c}-\mathrm{g}}$ & $12.4 \mathrm{def}$ & $13.4^{\mathrm{d}-\mathrm{h}}$ \\
\hline G8 & $81.1^{\mathrm{fg}}$ & $83.0^{\mathrm{a}-\mathrm{f}}$ & $82.0^{\text {cde }}$ & $31.1^{\text {ghı }}$ & $37.9^{b-f}$ & $34.5^{\mathrm{c}-\mathrm{g}}$ & $13.8^{h-j}$ & $12.3^{\text {e-f }}$ & $13.0^{\mathrm{h}}$ \\
\hline G9 & $81.8^{\mathrm{d}-\mathrm{f}}$ & $81.2^{\mathrm{k}}$ & $81.5^{\mathrm{e}-\mathrm{f}}$ & $37.4^{\mathrm{a}-\mathrm{d}}$ & $35.9^{\mathrm{c}-\mathrm{f}}$ & $36.6^{\mathrm{b}-\mathrm{e}}$ & $14.7^{\mathrm{bcd}}$ & $13.6^{\mathrm{a}}$ & $14.1^{\mathrm{a}}$ \\
\hline Pehlivan & $82.0^{\mathrm{d}-\mathrm{f}}$ & $83.3^{\mathrm{a}-\mathrm{d}}$ & $82.7^{a b c}$ & $36.9^{\mathrm{a}-\mathrm{e}}$ & $37.8^{b-f}$ & $37.3^{a b c}$ & $14.1^{\mathrm{e}-\mathrm{h}}$ & $12.7^{\mathrm{c}-\mathrm{f}}$ & $13.4^{\mathrm{d}-\mathrm{h}}$ \\
\hline G11 & $83.7^{\mathrm{a}}$ & $81.3^{\mathrm{j}-\mathrm{k}}$ & $82.5^{\mathrm{a}-\mathrm{d}}$ & $34.0^{\mathrm{b}-\mathrm{g}}$ & $35.3^{\text {def }}$ & $34.7^{\mathrm{c}-\mathrm{g}}$ & $13.4^{\mathrm{j}}$ & $13.1^{\mathrm{a}-\mathrm{d}}$ & $13.3^{\mathrm{f}-\mathrm{h}}$ \\
\hline G12 & $81.5^{\mathrm{e}-\mathrm{g}}$ & $81.6^{\mathrm{h}-\mathrm{k}}$ & $81.5^{\text {e-f }}$ & $39.4^{\mathrm{a}}$ & $39.1^{\mathrm{b}-\mathrm{e}}$ & $39.3^{\mathrm{ab}}$ & $13.8^{\mathrm{h}-\mathrm{j}}$ & $13.5^{a b}$ & $13.6^{\mathrm{b}-\mathrm{f}}$ \\
\hline G13 & $84.2^{\mathrm{a}}$ & $81.3^{\mathrm{k}}$ & $82.7^{a b c}$ & $32.3^{\mathrm{f}-1}$ & $38.8^{\mathrm{b}-\mathrm{e}}$ & $35.5^{\mathrm{c}-\mathrm{g}}$ & $15.0^{\mathrm{ab}}$ & $13.0^{\text {a-e }}$ & $14.0^{\mathrm{ab}}$ \\
\hline G14 & $84.0^{\mathrm{a}}$ & $82.2^{\mathrm{e}-\mathrm{k}}$ & $83.1^{\mathrm{a}}$ & $37.7^{a b c}$ & $33.1^{\mathrm{f}}$ & $35.4^{\mathrm{c}-\mathrm{g}}$ & $14.4^{\mathrm{c}-\mathrm{f}}$ & $12.1^{\mathrm{f}}$ & $13.3^{\mathrm{f}-\mathrm{h}}$ \\
\hline Cemre & $79.8^{\text {iij }}$ & $83.2^{\mathrm{a}-\mathrm{e}}$ & $81.5^{\text {e-f }}$ & $39.4^{\mathrm{a}}$ & $40.9^{a b c}$ & $40.1^{\mathrm{a}}$ & $14.4^{\mathrm{c}-\mathrm{f}}$ & $12.4^{\mathrm{def}}$ & $13.4^{\mathrm{d}-\mathrm{h}}$ \\
\hline G16 & $77.1^{\mathrm{mn}}$ & $81.5^{\mathrm{h}-\mathrm{k}}$ & $79.3^{j}$ & $29.8^{\text {hı }}$ & $40.8^{a b c}$ & $35.3^{\mathrm{c}-\mathrm{g}}$ & $14.5^{\mathrm{b}-\mathrm{e}}$ & $12.4 \mathrm{def}$ & $13.5^{\mathrm{c}-\mathrm{g}}$ \\
\hline G17 & $78.2^{\mathrm{kl}}$ & $83.2^{\text {a-e }}$ & $80.7^{\text {gh }}$ & $30.8^{\text {ghı }}$ & $35.9^{\mathrm{cdf}}$ & $33.4^{\mathrm{e}-\mathrm{g}}$ & $14.3^{\mathrm{c}-\mathrm{f}}$ & $13.1^{\mathrm{a}-\mathrm{d}}$ & $13.7^{\mathrm{a}-\mathrm{e}}$ \\
\hline G18 & $82.5^{\mathrm{cd}}$ & $83.8^{\mathrm{a}}$ & $83.1^{\mathrm{a}}$ & $33.6^{\mathrm{c}-\mathrm{h}}$ & $40.2^{b c d}$ & $36.9^{\mathrm{a}-\mathrm{d}}$ & $14.3^{\mathrm{c}-\mathrm{g}}$ & $12.8^{\mathrm{b}-\mathrm{f}}$ & $13.6^{\mathrm{b}-\mathrm{g}}$ \\
\hline G19 & $82.0^{\mathrm{df}}$ & $81.8^{\mathrm{g}-\mathrm{k}}$ & $81.9^{\mathrm{def}}$ & $37.9^{\mathrm{ab}}$ & $33.1^{\mathrm{f}}$ & $35.5^{\mathrm{c}-\mathrm{g}}$ & $14.7^{\mathrm{bcd}}$ & $12.6^{\mathrm{c}-\mathrm{f}}$ & $13.7^{\mathrm{b}-\mathrm{f}}$ \\
\hline Sagittario & $79.0^{\mathrm{jk}}$ & $81.5^{1-k}$ & $80.2^{\mathrm{h}}$ & $33.5^{\mathrm{c}-\mathrm{h}}$ & $39.6^{\mathrm{bcd}}$ & $36.6^{\mathrm{b}-\mathrm{f}}$ & $15.4^{\mathrm{a}}$ & $12.6^{\mathrm{c}-\mathrm{f}}$ & $14.0^{\mathrm{ab}}$ \\
\hline $\mathrm{G} 21$ & $83.3^{a b c}$ & $82.9^{\mathrm{a}-\mathrm{f}}$ & $83.1^{\mathrm{a}}$ & $33.5^{\mathrm{c}-\mathrm{h}}$ & $33.1^{\mathrm{f}}$ & $33.3^{\mathrm{e}-\mathrm{g}}$ & $13.5^{\mathrm{ij}}$ & $13.1^{\mathrm{a}-\mathrm{d}}$ & $13.3^{\mathrm{e}-\mathrm{h}}$ \\
\hline $\mathrm{G} 22$ & $80.8^{\mathrm{gh}}$ & $82.4^{\mathrm{d}-1}$ & $81.6^{\mathrm{e}-\mathrm{f}}$ & $34.3^{\mathrm{b}-\mathrm{g}}$ & $38.0^{\mathrm{b}-\mathrm{f}}$ & $36.1^{\mathrm{b}-\mathrm{f}}$ & $14.2^{\mathrm{d}-\mathrm{h}}$ & $12.5^{\mathrm{c}-\mathrm{f}}$ & $13.3^{\mathrm{d}-\mathrm{h}}$ \\
\hline $\mathrm{G} 23$ & $80.1^{\mathrm{h}}$ & $82.3^{d-j}$ & $81.2^{\mathrm{fg}}$ & $35.8^{\mathrm{a}-\mathrm{f}}$ & $36.5^{\mathrm{c}-\mathrm{f}}$ & $36.2^{\mathrm{b}-\mathrm{f}}$ & $14.5^{\mathrm{b}-\mathrm{e}}$ & $13.0^{\mathrm{a}-\mathrm{e}}$ & $13.7^{\mathrm{a}-\mathrm{d}}$ \\
\hline $\mathrm{G} 24$ & $82.6^{\mathrm{b}-\mathrm{d}}$ & $82.8^{\mathrm{a}-\mathrm{g}}$ & $82.7^{a b c}$ & $32.8^{\mathrm{e}-\mathrm{h}}$ & $42.5^{\mathrm{ab}}$ & $37.7^{a b c}$ & $14.3^{\mathrm{c}-\mathrm{g}}$ & $13.2^{a b c}$ & $13.8^{\mathrm{a}-\mathrm{d}}$ \\
\hline Adana-99 & $82.4^{\text {de }}$ & $83.6^{\mathrm{ab}}$ & $83.0^{\mathrm{ab}}$ & $31.3^{\mathrm{g} 1}$ & $36.0^{\mathrm{c}-\mathrm{f}}$ & $33.7^{\mathrm{d}-\mathrm{g}}$ & $13.9^{\mathrm{f}-1}$ & $12.5^{\mathrm{c}-\mathrm{f}}$ & $13.2^{\mathrm{g}-\mathrm{h}}$ \\
\hline Average & 81.0 & 82.4 & 81.7 & 33.8 & 37.5 & 35.7 & 14.2 & 12.8 & 13.5 \\
\hline Passed of check & 8 & 1 & 3 & 0 & 2 & 0 & 0 & 13 & 1 \\
\hline LSD (0.05) & $0.9 * *$ & $1.0 * *$ & $0.7 * *$ & $4.2 * *$ & $5.4 * *$ & $3.4 * *$ & $0.5^{* *}$ & $0.7^{*}$ & $0.5^{* *}$ \\
\hline
\end{tabular}

Av: Average

Cemre (40.1 g) cultivar took the first place in terms of thousand grain weight (Table 5). In a study carried out in bread wheat related to thousand grain weight, it was reported that thousand grain weight ranged between 29.6 and $53.8 \mathrm{~g}$, and the average was $40.7 \mathrm{~g}$. In this study, a thousand grain weight ranged between 32.8 and $40.1 \mathrm{~g}$. The average of the experiment was $35.7 \mathrm{~g}$. The results of the studies are similar (Panghal et al., 2019).

The average of protein content was $13.5 \%$. The highest protein content was obtained from G9 $(14.1 \%)$ line. In addition, the highest value in terms of protein content of the varieties used as standard in the study was obtained from Sagittario cultivar with $14.0 \%$ 
(Table 5). Karaman (2019), in his study based on precipitation conditions in bread wheat has reported that the average protein rate is $14.5 \%$. Also, there is a significant relationship between protein content and grain hardness. This has been reported to result from the strong interaction between carbohydrates and proteins. This result was confirmed by our study. (Table 5) (Preston, 1998; Dobraszczyk et al., 2002; Pasha et al., 2010; Baslar et al., 2012).

The average amount of zeleny sedimentation was $28.5 \mathrm{ml}$ and the $\mathrm{G} 12(35.1 \mathrm{ml})$ line have the highest sedimentation amount. In addition, it was determined that 4 lines had higher sedimentation amount than the highest standard variety (Sagittario; $31.6 \mathrm{ml}$ ) (Table 6). In the study of Koçak et al. (1992), the sedimentation value ranged from 18.8 to $43.8 \mathrm{ml}$, reported that the hereditary effect in sedimentation is higher than the effect of environmental factors. The obtained values are similar to our findings. It is emphasized that the amount of sedimentation is of great importance in determination of protein quality in bread wheat (Peterson et al., 1992).

Table 6. Zeleny sedimentation, wet gluten and grain hardness values

\begin{tabular}{|c|c|c|c|c|c|c|c|c|c|}
\hline \multirow{2}{*}{ Genotypes } & \multicolumn{3}{|c|}{$\mathrm{ZS}$ (ml) } & \multicolumn{3}{|c|}{ WG (\%) } & \multicolumn{3}{|c|}{ GH (PSI) } \\
\hline & 1. year & 2. year & Av. & 1. year & 2. year & Av. & 1. year & 2. year & Av. \\
\hline G1 & $24.0^{\text {def }}$ & $34.5 \mathrm{bcd}$ & $29.3^{\mathrm{d}-\mathrm{g}}$ & $29.9^{\mathrm{e}-\mathrm{h}}$ & $29.4^{\mathrm{a}-\mathrm{f}}$ & $29.6^{\mathrm{d}-\mathrm{g}}$ & $52.1^{\mathrm{h}}$ & $47.0^{\mathrm{gh}}$ & $49.6^{\mathrm{h}}$ \\
\hline $\mathrm{G} 2$ & $36.5^{\mathrm{a}}$ & $29.0^{\mathrm{h}-\mathrm{k}}$ & $32.8^{a b c}$ & $29.4^{\mathrm{g}-\mathrm{h}}$ & $30.1^{\mathrm{a}-\mathrm{e}}$ & $29.8^{\mathrm{d}-\mathrm{g}}$ & $56.2^{\mathrm{d}-\mathrm{g}}$ & 51.2 def & 53.7 def \\
\hline G3 & $28.5^{\text {b-e }}$ & $31.0^{\mathrm{e}-\mathrm{h}}$ & $29.8^{c-f}$ & $32.7^{b c}$ & $29.9^{\mathrm{a}-\mathrm{e}}$ & $31.3^{\mathrm{abc}}$ & $57.2^{\mathrm{c}-\mathrm{e}}$ & $52.2^{\mathrm{c}-\mathrm{e}}$ & $54.7^{\mathrm{cd}}$ \\
\hline G4 & $23.5^{\mathrm{ef}}$ & $31.7^{\mathrm{e}-\mathrm{g}}$ & $27.6^{\mathrm{e}-\mathrm{k}}$ & $29.9^{\text {e-h }}$ & $29.6^{\mathrm{a}-\mathrm{f}}$ & $29.7^{\mathrm{d}-\mathrm{g}}$ & $49.0^{1 \mathrm{ij}}$ & $43.9^{\mathrm{h}}$ & $46.5^{1}$ \\
\hline Nurkent & $25.5^{\mathrm{c}-\mathrm{f}}$ & $28.3^{\mathrm{e}-\mathrm{k}}$ & $26.9^{\mathrm{f}-\mathrm{k}}$ & $30.7^{\mathrm{def}}$ & $28.3^{\mathrm{c}-\mathrm{g}}$ & $29.5^{\mathrm{d}-\mathrm{g}}$ & $48.8^{\mathrm{ijj}}$ & $43.7^{\mathrm{h}}$ & $46.3^{1}$ \\
\hline G6 & $24.0^{\mathrm{def}}$ & $26.7^{\mathrm{k}}$ & $25.3^{\mathrm{j}-\mathrm{k}}$ & $30.7^{\mathrm{d}-\mathrm{g}}$ & $28.1^{\mathrm{c}-\mathrm{g}}$ & $29.4^{\text {efg }}$ & $56.2^{\mathrm{d}-\mathrm{g}}$ & $51.2^{\mathrm{def}}$ & $53.7^{\mathrm{def}}$ \\
\hline G7 & $35.0^{\mathrm{a}}$ & $33.3^{\mathrm{b}-\mathrm{e}}$ & $34.2^{\mathrm{ab}}$ & $31.5^{\mathrm{cd}}$ & $27.7^{\mathrm{d}-\mathrm{g}}$ & $29.6^{\mathrm{d}-\mathrm{g}}$ & $54.1^{\mathrm{e}-\mathrm{h}}$ & $49.1^{\mathrm{efg}}$ & $51.6^{\mathrm{fgh}}$ \\
\hline G8 & $23.5^{\text {ef }}$ & $30.7^{\mathrm{f}-1}$ & $27.1^{\mathrm{f}-\mathrm{k}}$ & $29.6^{\mathrm{f}-\mathrm{h}}$ & $27.6^{\text {efg }}$ & $28.6^{\mathrm{g}}$ & $59.0 \mathrm{bcd}$ & $54.0^{\mathrm{bcd}}$ & $56.5^{\mathrm{bc}}$ \\
\hline G9 & $30.3^{\mathrm{a}-\mathrm{d}}$ & $29.7^{\mathrm{g}-\mathrm{j}}$ & $30.0^{\mathrm{c}-\mathrm{f}}$ & $31.6^{b-d}$ & $31.7^{\mathrm{a}}$ & $31.6^{\mathrm{a}}$ & $52.7^{\mathrm{h}}$ & $47.7^{\mathrm{g}}$ & $50.2^{\mathrm{gh}}$ \\
\hline Pehlivan & 23.5 ef & $28.8^{\mathrm{h}-\mathrm{k}}$ & $26.2^{\mathrm{g}-\mathrm{k}}$ & $30.2^{\mathrm{e}-\mathrm{h}}$ & $28.5^{\mathrm{c}-\mathrm{g}}$ & $29.3^{\mathrm{efg}}$ & $54.8^{\mathrm{e}-\mathrm{h}}$ & $49.7^{\mathrm{efg}}$ & $52.3^{\mathrm{e}-\mathrm{g}}$ \\
\hline G11 & $32.5^{\mathrm{ab}}$ & $33.0^{\mathrm{c}-\mathrm{f}}$ & $32.8^{a b c}$ & $28.0^{1}$ & $30.2^{\mathrm{a}-\mathrm{d}}$ & $29.1^{\mathrm{efg}}$ & $49.1^{\mathrm{iij}}$ & $44.0^{\mathrm{hr}}$ & $46.6^{1}$ \\
\hline G12 & $35.0^{\mathrm{a}}$ & $35.2^{a b c}$ & $35.1^{\mathrm{a}}$ & $29.2^{\mathrm{hl}}$ & $31.4^{\mathrm{ab}}$ & $30.3^{a-f}$ & $60.7^{\mathrm{ab}}$ & $55.7^{a b}$ & $58.2^{\mathrm{b}}$ \\
\hline G13 & $25.5^{\mathrm{c}-\mathrm{f}}$ & $28.7^{\mathrm{h}-\mathrm{k}}$ & $27.1^{\mathrm{f}-\mathrm{k}}$ & $32.8^{\mathrm{ab}}$ & $29.6^{\mathrm{a}-\mathrm{f}}$ & $31.2^{\mathrm{abc}}$ & $47.6^{j}$ & $42.6^{1}$ & $45.1^{1}$ \\
\hline G14 & $22.0^{f}$ & $27.0^{\mathrm{k}}$ & $24.5^{\mathrm{k}}$ & $31.5^{\mathrm{cd}}$ & $26.5^{\mathrm{g}}$ & $29.0^{\mathrm{fg}}$ & $47.7^{\mathrm{j}}$ & $42.6^{1}$ & $45.2^{1}$ \\
\hline Cemre & $24.0^{\mathrm{def}}$ & $27.8^{\mathrm{jk}}$ & $25.9^{\mathrm{g}-\mathrm{k}}$ & $32.4^{\mathrm{bc}}$ & $27.8^{\mathrm{d}-\mathrm{g}}$ & $30.1^{\text {b-f }}$ & $63.8^{a}$ & $58.8^{\mathrm{a}}$ & $61.3^{\mathrm{a}}$ \\
\hline G16 & $24.5^{\mathrm{def}}$ & $27.0^{\mathrm{k}}$ & $25.8^{h-k}$ & $31.1^{\text {de }}$ & $27.6^{\mathrm{e}-\mathrm{g}}$ & $29.3^{\mathrm{e}-\mathrm{g}}$ & $53.5^{\mathrm{gh}}$ & $48.9^{\mathrm{efg}}$ & $51.2^{\mathrm{gh}}$ \\
\hline G17 & $31.0^{\mathrm{abc}}$ & $27.0^{\mathrm{k}}$ & $29.0^{\mathrm{d}-1}$ & 30.8 def & $28.3^{\mathrm{c}-\mathrm{g}}$ & $29.5^{\mathrm{d}-\mathrm{g}}$ & $56.4^{\mathrm{d}-\mathrm{g}}$ & $51.4^{\mathrm{def}}$ & $53.9^{\text {def }}$ \\
\hline G18 & $24.0^{\mathrm{def}}$ & $37.6^{\mathrm{a}}$ & $30.8^{b-e}$ & $32.6^{\mathrm{bc}}$ & $29.1^{\text {b-g }}$ & $30.8^{\mathrm{a}-\mathrm{d}}$ & $53.7^{\text {fh }}$ & $48.7 \mathrm{fg}$ & $51.2^{\mathrm{gh}}$ \\
\hline G19 & $23.0^{\mathrm{ef}}$ & $29.0^{\mathrm{h}-\mathrm{k}}$ & $26.0^{\mathrm{g}-\mathrm{k}}$ & $31.6^{\mathrm{bcd}}$ & $28.3^{\mathrm{c}-\mathrm{g}}$ & $29.9^{\mathrm{c}-\mathrm{g}}$ & $48.9^{\mathrm{ijj}}$ & $43.9^{\mathrm{h}}$ & $46.4^{1}$ \\
\hline Sagittario & $27.5^{\text {b-f }}$ & $35.7^{a b}$ & $31.6^{\mathrm{b}-\mathrm{d}}$ & $34.1^{\mathrm{a}}$ & $29.0^{b-g}$ & $31.5^{\mathrm{a}}$ & $60.3^{b c}$ & $55.3^{b c}$ & $57.8^{b}$ \\
\hline $\mathrm{G} 21$ & $24.5^{\mathrm{def}}$ & $28.0^{\mathrm{jk}}$ & $26.3^{\mathrm{g}-\mathrm{k}}$ & $29.5^{\mathrm{f}-\mathrm{h}}$ & $30.4^{\mathrm{abc}}$ & $30.0^{\mathrm{b}-\mathrm{g}}$ & $48.2^{\mathrm{j}}$ & $43.2^{1}$ & $45.7^{1}$ \\
\hline $\mathrm{G} 22$ & $24.0^{\mathrm{def}}$ & $27.3^{\mathrm{jk}}$ & $25.7^{1-k}$ & $30.5^{\mathrm{d}-\mathrm{g}}$ & $27.5^{\mathrm{e}-\mathrm{g}}$ & $29.0^{\mathrm{fg}}$ & $48.4^{\mathrm{j}}$ & $42.4^{1}$ & $45.4^{1}$ \\
\hline $\mathrm{G} 23$ & $26.5^{b-f}$ & $27.0^{\mathrm{k}}$ & $26.8^{\mathrm{f}-\mathrm{k}}$ & $31.6^{\mathrm{bcd}}$ & $31.1^{\mathrm{ab}}$ & $31.4^{\mathrm{ab}}$ & $56.3^{\mathrm{d}-\mathrm{g}}$ & $51.3^{\mathrm{def}}$ & $53.8^{\mathrm{def}}$ \\
\hline $\mathrm{G} 24$ & $25.0^{\mathrm{c}-\mathrm{f}}$ & $31.7^{\mathrm{e}-\mathrm{g}}$ & $28.3^{\mathrm{d}-\mathrm{j}}$ & $31.0^{\text {de }}$ & $30.0^{\mathrm{a}-\mathrm{e}}$ & $30.5^{\mathrm{a}-\mathrm{e}}$ & $57.1^{\mathrm{c}-\mathrm{f}}$ & $52.1^{\mathrm{c}-\mathrm{f}}$ & $54.6^{\text {cde }}$ \\
\hline Adana-99 & $26.0^{c-f}$ & $32.2^{\text {def }}$ & $29.1^{\mathrm{d}-\mathrm{h}}$ & $30.6^{\mathrm{d}-\mathrm{g}}$ & $27.3^{\mathrm{fg}}$ & $28.9^{\mathrm{f}-\mathrm{g}}$ & $59.4^{\text {bcd }}$ & $54.4^{\mathrm{b}-\mathrm{d}}$ & $56.9^{\mathrm{bc}}$ \\
\hline Average & 26.8 & 30.3 & 28.5 & 30.9 & 29.0 & 30.0 & 54.0 & 49.0 & 51.5 \\
\hline Passed of check & 7 & 1 & 4 & 0 & 12 & 1 & 0 & 0 & 0 \\
\hline LSD (0.05) & $6.4^{* *}$ & $2.4^{* *}$ & $3.4 * *$ & $1.3 * *$ & $2.6 * *$ & $1.4 * *$ & $3.4 * *$ & $3.4 * *$ & $2.4 * *$ \\
\hline
\end{tabular}


The mean of wet gluten ratio was found to be $30.0 \%$, while G9 (31.6\%) and Sagittario $(31.5 \%)$ had the highest value by sharing the same group (Table 6). It has been reported that wet gluten ratio in bread wheat is important and the amount of gluten formed as a result of swelling of gliadin and gluten, which are subcomponents of protein, by absorbing water, is affected significantly by environmental conditions. The researchers reported that wet gluten content varied between 11.3 and 45.2\% (Keçeli et al., 2017).

In the study, the mean experiment value of grain hardness was found to be 51.5 (PSI). Nurkent (46.3 PSI) had the hardest grain type and Cemre (61.3 PSI) had the softest grain type. In the interpretation of the grain hardness parameter, it is interpreted that the grain hardness increases with decreasing PSI value and decreases with increasing PSI value (Sahin et al., 2017). According to this, G13 (45.1) was found to be the genotype with the highest grain hardness. G4 (46.5), Nurkent (46.3), G11 (46.6), G14 (45.2), G19 (46.4), G21 (45.7) and G22 (45.4) lines were also good genotypes for grain hardness (Table 6). Because grain hardness is positively associated with protein (Table 7).

Grain hardness (PSI) parameter is important in bread wheat. Especially when flour is obtained from hard wheat, starch damage is more than soft wheat. It was emphasized that this was not preferred by bakers. Also, dough made from a high damaged starch content flour was reported to have more water-holding capacity (Khan and Shewry, 2009; Sahin et al., 2017).

\section{Correlation analysis}

Respect to correlation analysis, there was a positive correlation between grain yield and test weight and a negative correlation with wet gluten ratio. While test weight increased, wet gluten ratio and grain hardness (PSI) values were found to decrease. Thousand grain weight was found to be inversely related to protein content and wet gluten ratio. In addition, the content of protein and wet gluten was found to be inversely related to the amount of zeleny sedimentation (Table 7). This situation is contrary to the literature. Because many researchers have reported that the amount of zeleny sedimentation positively correlated with protein content and wet gluten ratio (Surma et al., 2012; Keceli et al., 2017).

Table 7. Correlation coefficients and significance levels between parameters

\begin{tabular}{c|c|c|c|c|c|c|c|c}
\hline Features & GY & TW & TGW & PR & ZS & WG & GH & HT \\
\hline TW & $0.325 * *$ & & & & & & & \\
TGW & 0.096 & $0.227 * *$ & & & & & & \\
PR & -0.149 & $-0.432 * *$ & $-0.329 * *$ & & & & & \\
ZS & -0.146 & -0.070 & 0.256 & $-0.284 * *$ & & & & \\
WG & $-0.201 *$ & $-0.364 * *$ & $-0.185 *$ & $0.860 * *$ & $-0.165 *$ & & & \\
GH & 0.037 & $-0.274 * *$ & -0.047 & $0.397 * *$ & -0.076 & $0.323 * *$ & & \\
HT & -0.091 & $-0.394 * *$ & $-0.368 * *$ & $0.703 * *$ & $-0.356 * *$ & $0.459 * *$ & $0.565 * *$ & \\
PH & 0.093 & -0.105 & -0.147 & $0.540 * *$ & $-0.342 * *$ & $0.295^{* *}$ & $0.350 * *$ & $0.629 * *$ \\
\hline
\end{tabular}

$* * 1 \%, * 5 \%$ important 
This may be due to the low protein quality despite the high protein content of the genotypes or vice versa. Additionally, grain hardness (PSI) was positively correlated with heading time and plant height. Kılıç et al. (2014) obtained similar results in their study.

\section{Conclusion}

In the study, early heading cultivars were found to be prominent in terms of grain yield and test weight. However, it was determined that the same situation was not observed in terms of quality parameters. It was found that there was a positive correlation between grain yield and test weight and a negative correlation with wet gluten. There was a negative correlation between grain hardness (PSI) and test weight and a positive correlation between plant height and heading time. G1, G14, G19, G24 for grain yield, G14, G18, G21 for test weight; G9 for protein ratio and wet gluten amount, G2, G7, G11, G12, for zeleny sedimentation outperformed all standards. In the study, it is determined that the lines which are superior to standard varieties in terms of one or a few features should be taken into consideration in grain yield or qualityoriented breeding studies. It was concluded that G19 and G24, which have superior features, can be registered candidates. In the future, if collaboration with modern breeding (molecular) is done, genotypes of superior will be studied and the results will shed light on future research.

\section{REFERENCES}

[1] Aktas, H. (2017): Evaluation of some barley (Hordeum vulgare L.) cultivars commonly cultivated in Turkey under supplemented irrigation and rainfall conditions. - J. Tekirdag Agr. Fac. 14(03): 86-97.

[2] Anonymous (1982): ICC-Standard No: 115/1. - International Association for Cereal Chemistry, Vienna.

[3] Anonymous (1990): Approved Methods of the American Association of Cereal Chemistry, USA. - AACC, Washington, DC.

[4] Anonymous (1994): ICC - Standard No: 155/1. - International Association for Cereal Chemistry, Vienna.

[5] Anonymous (2000): AACC. Approved Methods. Vol. 2. $8^{\text {th }}$ Ed. - Repr. American Association of Cereal Chemists, St. Paul, MA.

[6] Anonymous (2013): GAP International Agricultural Research and Training Center. - Soil Analysis Laboratory, Diyarbakir.

[7] Barutcular, C., Yildirim, M., Koç, M., Akinci, C., Toptas, I., Albayrak, O., Tanrikulu, A., EL Sabagh, A. (2016): Evaluation of SPAD chlorophyll in spring wheat genotypes under different environments. - Fresenius Env. Bul. 25(4): 1258-1266.

[8] Baslar, M., Kalkan, F., Kara, M., Ertugay, M. F. (2012): Correlation between the protein content and mechanical features of wheat. - J. Agr. and Forest. 36: 601-607.

[9] Caierao, E. (2006): Brazilian society of plant breeding. Printed in brazil effect of induced lodging on grain yield and quality of brewing barley. - Crop Breed. Appl. Biotech. 6: 215-221.

[10] Civgin, İ. (2016): The factors that affect plant domestication process in the fertile crescent: climate, natural habitat and cross-cultural encounters (11000-7000 BCE.) Mehmet Akif Ersoy Univ. - J. of the Ins. of Soc. Sci. 8(17): 463-488.

[11] Dobraszczyk, B. J., Whitworth, M. B., Vincent, J. F. V., Khan, A. A. (2002): Single kernel wheat grain hardness and fracture features in relation to density and the modelling of fracture in wheat endosperm. - J. Cereal Sci. 35: 245-263. 
[12] Gomez, K. A., Gomez, A. A. (1984): Statistical Procedures for Agricultural Research. 2nd. Ed. - John Willey and Sons, Inc. New York.

[13] Gooding, M. J., Ellis, R. H., Shewry, P. R., Schofield, J. D. (2003): Effects of restricted water availability and increased temperature on the grain filling, drying and quality of winter wheat. - J. Cereal Sci. 37: 295-309.

[14] Grausgruber, H., Oberforster, M., Werteker, M., Ruckenbauer, P., Vollmann, J. (2000): Stability of quality traits in Austrian-grown winter wheats. - Field Crop. Res. 66: 257267.

[15] Karaman, M. (2019): Evaluation of bread wheat genotypes in irrigated and rainfed conditions using biplot analysis. - Applied Ecol. Env. Res. 17(1): 1431-1450.

[16] Keçeli, A., Kaplan Evlice, A., Pehlivan, A., Şanal, T., Karaca, K., Külen, S., Seis Subaşı, A., Salantur, A. (2017): Investigation of the relationship between zeleny sedimentation analysis and other quality parameters in bread wheat (Triticum aestivum L.). Kahramanmaraş Sütçü İmam Univ. - J. Natur. Sci. 20(Special Issue): 292-296.

[17] Kendal, E., Sener, O. (2015): Examination of genotype $\times$ environment interactions by GGE biplot analysis in spring durum wheat. - Indian J. Gen. Plant Breed. 75(3): 341-348.

[18] Khan, K., Shewry, P. R. (2009): Criteria of Wheat and Flour Quality. - In: Gordon, R., Carson, N., Edwards, M. (eds.) Wheat and Chemistry. Fourth Ed. Chap. 4. AACC International Inc., St. Paul.

[19] Kilic, H., Kendal, E., Aktas, H., Tekdal, S. (2014): Assessment of advanced bread wheat lines for yield and some quality traits at different environment. - Iğdir Univ. J. Inst. Sci. Tech. 4(4): 87-95.

[20] Kiseleva, A. A., Salina, E. A. (2018): Genetic regulation of common wheat heading time. - Russian J. Genetic. 54: 375-388

[21] Kizilgeci, F. (2019): Physiological, agronomical and quality response of bread wheat to phosphorus application under dryland condition. - Applied Ecol. Env. Res. 17(2): 19791987.

[22] Kocak, N., Atli, A., Karababa, E., Tuncer, T. (1992): Macar-Yugoslav (MAYEB) A research on the quality properties of Hungarian Yugoslavia bread wheat varieties. - J. Field Crop. Cent. Res. Inst. 1(1): 27-45.

[23] Morgounov, A., Keser, M., Kan, M., Küçükçongar, M., Özdemir, F., Gummanow Muminjanov, H., Zuev, E., Qualset, C. O. (2016): Wheat landraces currently grown in Turkey: distribution, diversity and use. - Crop Sci. 56: 1-13.

[24] Panghal, A., Chhikara, N., Khatkar, B. S. (2019): Characterisation of Indian wheat varieties for chapatti (flat bread) quality. - J. Saudi Soc. Agr. Sci. 18(1): 107-111.

[25] Pasha, I., Anjum, F. M., Morris, C. F. (2010): Grain hardness: a major determinant of wheat quality. - Food Sci. Tech. Inter. 16: 511-522.

[26] Peterson, C. J., Graybosch, R. A., Baenziger, P. S., Grombacher, A. W. (1992): Genotype and environment effects on quality characteristics of hard red winter wheat. - Crop Sci. 32: 98-103.

[27] Preston, K. R. (1998): Protein-Carbohydrate Interactions. - In: Hamer, R. J., Hoseney, R. C. (eds.) Interactions: The Keys to Cereal Quality. AACCI, St. Paul, MN, pp. 83-91.

[28] Souza, E., Martin, J. M., Guttieri, M. J., O’Brien, K. M., Habernicht, D. K., Lanning, S. P., McLean, R., Carlson, G. R., Talbert, L. E. (2004): Influence of genotype, environment, and nitrogen management on spring wheat quality. - Crop Sci. 44: 425-432.

[29] Sahin, M., Gocmen Akcacik, A., Aydogan, S., Hamzaoglu, S., Demir, B., Yakişir, E. (2017): Investigation of the relationship between zeleny sedimentation and yield and some quality traits in winter bread wheat varieties. - J. Bahri Dag. Crop Res. 6(1): 10-21.

[30] Surma, M., Adamski, T., Banaszak, Z., Kaczmarek, Z., Kuczynska, H., Majcher, M., Ługowska, B., Obuchowski, W., Salmanowicz, B., Krystkowiak, K. (2012): Effect of genotype, environment and their interaction on quality parameters of wheat breeding lines of diverse grain hardness. - Plant Product. Sci. 15(3): 192-203. 
[31] Tekdal, S., Yildirim, M. (2017): Relation of physiological and morphological parameters with heat stress in some durum wheat genotypes. - Turkish J. Nat. Sci. 6(2): 73.

[32] TSI (2018): Turkish Statistical Institute. - Crop Production Statistics, www.tuik.gov.tr. 